\title{
Clinical Evaluation Methods of Oral Cavity Hygiene of Patients with Drug-Compensated Diabetes Mellitus Using Removable Partial Acrylic Prosthesis
}

\author{
Shevkunova Natalia Alekseevna* \\ Department of Prosthetic Dentistry, Federal State Budgetary Educational Institution of Higher Professional Education "Izhevsk State Medical \\ Academy" of Ministry of Health of Russia, Russia
}

Received: 眥 March 09, 2018; Published: 㘹 March 16, 2018

*Corresponding author: Shevkunova NA, Department of Prosthetic Dentistry, Federal State Budgetary Educational Institution of Higher Professional Education "Izhevsk State Medical Academy" of Ministry of Health of Russia, Russia

\begin{abstract}
Summary
The influence of hygiene products on the state of the oral cavity and dentures in 63 patients with type 2 diabetes using partial removable acrylic prostheses was studied. The results of the study showed that individual oral hygiene with the use of rinses and the care for removable dentures with the use of cleansing tablets contributed to the increase of the index of effectiveness of oral hygiene RNR (Podshadley, Haley, 1968) and removable dentures (Ambjörnsen E.e.a.). The conducted measures reduced inflammatory phenomena in the tissues of the prosthetic bed in diabetic patients, which provided stable clinical results in long-term follow-up [1-8].
\end{abstract}

Keywords: Diabetes Mellitus type 2; The efficiency of hygiene of the oral cavity; Hygienic condition of removable dentures

\section{The Relevance of the Study}

The annual increase in the number of diabetics determines the medico-social and clinical significance of this problem, including dentistry. Medically compensated diabetes mellitus type 2 (SD2) is diagnosed in patients older than 40 years, with $51 \%$ - 98\% observed inflammatory lesions of periodontal tissues, bone resorption and as a consequence a high percentage of complete or partial edentulous [9-12]. Partial absence of teeth in case of untimely orthopedic treatment leads to morphological and functional restructuring of the entire dental system, causing inflammatory and destructive changes in the mucous membrane of the oral cavity and periodontal tissues [1,10]. Additional difficulties in orthopedic dental rehabilitation of this category of patients cause increased formation of plaque, reduced resistance to infection, resistance to yeast-like fungi, which is a risk factor in the development of complications and increased level of glycemia by increasing the body's resistance to insulin, causing deterioration during diabetes $[4,6,9]$. In this regard, the role of individual oral and prosthetic hygiene in orthopedic treatment of such patients and the expediency of using means that provide antibacterial and anti-inflammatory effect [5-9] increases.

\section{Purpose}

To study the effect of hygiene products on the condition of the oral cavity and dentures of patients with SD2 in the treatment of partial removable acrylic prostheses.

\section{Materials and Methods}

A randomized clinical examination was conducted of 63 patients diagnosed with type 2 diabetes mellitus (38 women and 25 men) aged from 48 to 67 years and duration of the disease was $6,8 \pm 1,7$ years. Control of blood sugar level was carried out by the patients individual picometre, the average was $8,2-1,2 \mathrm{mmol} / \mathrm{l}$. To determine the awareness of the necessary hygienic measures to care for the oral cavity and prostheses a survey of all respondents was held. In the first phase of the study before orthopedic treatment, all patients underwent professional oral hygiene - removal of supraand subgingival dental plaque by ultrasound using the apparatus "Skeller" and "Piezon Master". A comprehensive study of dental status included clinical and radiological examination methods, determination of oral hygiene by the index of hygiene effectiveness 
(Podshadley, Haley, 1968) after staining with liquid to determine dental plaque.

In the absence of an index tooth the next one was examined, within the same group of teeth. Artificial crowns and parts of fixed prostheses were examined as well as teeth. To assess the hygienic condition of removable partial acrylic prosthesis was used a modified technique Ambjörnsen E.e.a. (1982). All respondents had defects of dentition, the diagnosis of which was carried out according to the "Protocol of management of patients with partial absence of teeth (partial secondary adentia)" [3]. In the survey, we enrolled patients with the absence of more than 6 teeth on one jaw. The average number of missing teeth was $8,2 \pm 1,1$. The exclusion criterion from the study of 9 patients with SD2 was the presence of complete secondary edentulous and the absence of more than 10 teeth on one of the jaws.

Orthopedic treatment of defects in the dentition of the upper or lower jaw was carried out with the use of removable plate prostheses with clammer fixation system, manufactured in dental laboratory of the Republican dental clinic of Izhevsk from basic plastic of one manufacturer. All patients were under medical supervision during the month, with regular check-ups once a week. During the observation period, patients with diabetes of the experimental group (31 people) performed the complex of therapeutic and prophylactic measures, which included:

i. Cleaning of teeth with the help of a conventional manual toothbrush using therapeutic and prophylactic toothpastes in the morning and in the evening;

ii. The use of balms-rinses for the oral cavity of plant origin after meals;

iii. Use of a toothbrush to clean dentures

iv. Treatment of removable plate dentures with special cleaning tablets daily in accordance with the instructions of the manufacturer.

At the same time, patients with diabetes in the comparison group (32 people) continued to use previously available oral hygiene products and comply with traditional recommendations for the hygiene of removable orthopedic structures. Parametric and nonparametric methods were used for statistical processing of the obtained results. In the case of deviation from the normal distribution, the nonparametric Wilcoxon-Mann -Whitney criterion was used to compare the data. When normality of the data distribution, we used t-criterion of Student. Statistical analysis included the calculation of arithmetic mean (M), arithmetic mean error $(\mathrm{m})$, and probability of differences (p). Differences were evaluated as significant at $\mathrm{p}<0,05$, analysis of the results was performed using the software package Microsoft Office Excel 2007.

\section{Results and Discussion}

The results of the survey showed insufficient awareness of patients about hygiene skills in oral care and dentures. The third part of the respondents (19 people, 30,1\%) cleaned teeth twice a day, more than half (39 people, $60,9 \%$ ) took care of teeth once a day, all respondents cleaned teeth less than 2 minutes and changed the toothbrush as the previous was worn out. $77,4 \%$ of patients did not receive recommendations from the dentist on the peculiarities of oral care, and $89,9 \%$ did not use additional hygiene products. In the study the index of efficiency of hygiene of an oral cavity (PHP) before orthopedic treatment in 20,8\% of surveyed patients with SD2 indicators was $1,4 \pm 0,1$ and was assessed as satisfactory, $79,2 \%$ of the performance of the PHP was evaluated as unsatisfactory and was $1,7 \pm 0,2$. A month after the orthopedic rehabilitation with partial removable plate prostheses with clammer fixation system, the indices of PHP in patients of the experimental group were $0,3 \pm 0,1$ and were evaluated as good, while the similar index in patients with diabetes in the comparison group was $1,9 \pm 0,2(t=3,34 ; p<0,01)$ corresponding to the poor efficiency of oral hygiene.

The hygienic state of the removable laminar dentures, defined according to the modified method of E. Ambjörnsen e. a. (1982) also distinguished with patients experienced and the comparative groups and was $1,4 \pm 0.25$ and $2,3 \pm 0,1$ respectively $(p<0,05)$. Index estimation of the number of prosthetic plaque revealed that in patients of the comparison group the greatest number $(4,01 \pm 0,9)$ was found on the inner surface of the bases of the lower dentures in the projection of the chewing group of teeth, on the inner surface of the bases of the upper dentures along the transition of the hard palate into the soft palate $(3,1 \pm 0,6)$ and less on the inner surface of the bases in the frontal sections of the upper and lower dentures $(1,3 \pm 0,3)$. In the experimental group, the prosthetic plaque was present on the inner surface of the upper jaw prosthesis bases in the projection of the chewing group of teeth $(1,2 \pm 0,3 ; \mathrm{P}<0,01)$.

The obtained results indicate that with tooth loss of patients with type 2 diabetes mellitus, the effectiveness of oral hygiene is at a satisfactory and unsatisfactory level. Orthopedic dental rehabilitation of such patients and the use of special funds to care for the oral cavity showed improvement of performance indicators of hygiene in 2 times in comparison with the similar indicators in the traditional methods of hygiene. The use of cleaning tablets and special brushes in the care of removable plate prostheses can improve the hygienic condition of removable plate prostheses by 1,7 times.

\section{Conclusion}

Thus, a comparative analysis of the hygienic condition of the oral cavity and removable plate prostheses of patients with SD2 of experimental and comparative groups showed that the daily use of therapeutic and prophylactic toothpastes and rinses of plant origin 
allows maintaining a good level of oral hygiene even in the presence of factors of dental plaque retention-removable acrylic prostheses with clammer fixation. Hygienic care of removable plate prostheses with the use of cleansing tablets in diabetes mellitus is a preventive measure reducing the risk of possible complications, which contributes to successful adaptation and increases the duration of use of prostheses. It is important to inform patients with SD2 about special care products for oral cavity and dentures in orthopedic dental rehabilitation, as inflammation in the oral cavity adversely affects the level of glycemia and affects the general condition of the body.

\section{References}

1. Verkhovsky AE, Abolmasov NN, Fedosov EA, Asoskova OV (2015) Comparative analysis of the results of examination and treatment of patients using acrylic removable dentures. Russian dental journal 6: 1317.

2. Bykova NI, Skorikova LA (2013) Influence of oral hygiene products on biochemical parameters of oral fluid in partial edentulous. Kuban scientific medical Herald 6(141): 49-55.

3. Gost R (2008) The Protocol of management of patients. Partial absence of teeth (partial secondary adentia). M Publishing house of standards.

4. Zhirnova AI, Shcherbakov AS, Chervinets UV (2015) Clinical features of oral tissues in patients with diabetes undergoing orthopedic treatment of dental prostheses of various structural materials. Modern problems of science and education 4: 43-47.

5. Zhulev EN, Tiunova NV, Levin IA (2015) Increase of efficiency of hygiene of dentures with the use COREGA TABS of patients with stomalgia. Institute of dentistry 3(68): 56-58.

6. Kerimov RA (2013) The results of clinical studies in the dental rehabilitation of patients with type 2 diabetes. The light of medicine and biology 4: 27-29.

7. Makeeva MK (2011) The use of mouthwashes for the oral cavity in a complex of individual hygiene care. Institute of dentistry 3(52): 74-75.

8. Ponomareva NA, Kurakina NV (2007) The antibacterial properties of phytoparasitica to care for the oral cavity from protezionecivile of elderly and senile age. Russian medical-biological Bulletin them academician I. P. Pavlov 4: 116-120.

9. Prozorova NV, Mamikin KE (2015) Evaluation of the effect of oral hygiene on the periodontal status of patients with diabetes mellitus. Bulletin of the Novgorod state University 2(85): 86-88.

10. Furtsev TV, Saleeva GT (2009) Neediness and the state of orthopaedic care of patients with diabetes mellitus- Kazan: 115-117.

11. (1999) American Academy of Periodontology. Diabetes and periodontal desease (position paper). 70: 935-949.

12. Iacopino AM (2001) Periodontitis and diabetes interrelationships: Role of inflamation. Ann periodontal 6: 125-137.
(C) Commons Attribution 4.0 License

To Submit Your Article Click Here: Submit Article

DOI: 10.32474/MADOHC.2018.01.000120

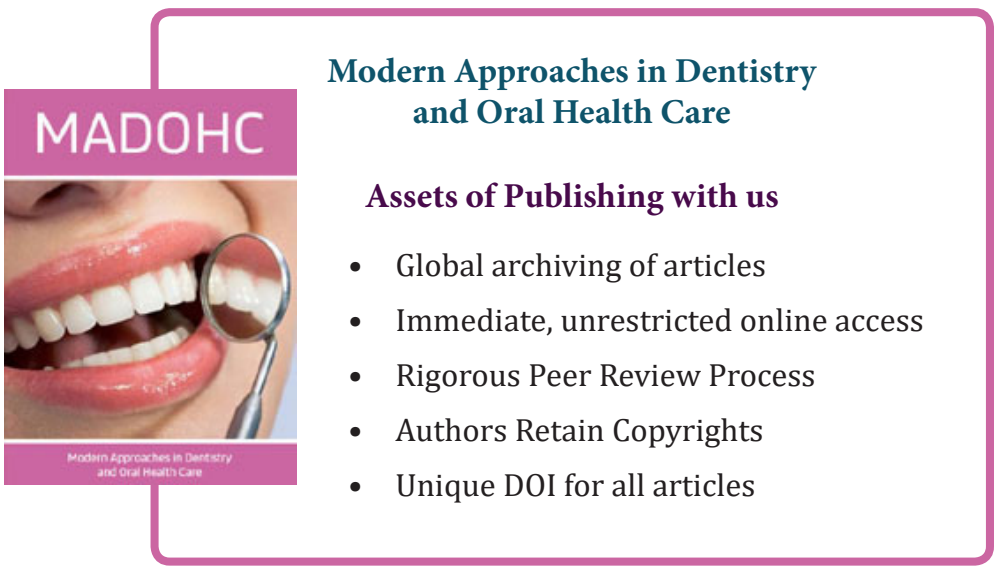

